\title{
ESTUDO EXPERIMENTAL DA RELAÇÃO DA DESSULFURAÇÃO EM LEITO FLUIDIZADO BORBULHANTE COM AS CONDIÇÕES FLUIDODINÂMICAS
}

\author{
${ }^{12}$ G.M. FAÉ GOMES, ${ }^{12}$ L. DALLA ZEN, ${ }^{1}$ C. PHILIPPSEN, ${ }^{1}$ E.K. BARD, ${ }^{1}$ G. SOUZA \\ ${ }^{1}$ Fundação de Ciência e Tecnologia do Rio Grande do Sul (CIENTEC), Departamento de \\ Engenharia de Processos \\ ${ }^{2}$ Universidade do Vale do Rio dos Sinos (UNISINOS), Escola de Engenharia \\ E-mail para contato: gabriel-gomes@cientec.rs.gov.br
}

\begin{abstract}
RESUMO - Algumas questões operacionais que envolvem a dessulfuração em leito fluidizado ainda necessitam do desenvolvimento de um maior conhecimento. Dentre elas, destaca-se a relação existente entre as características fluidodinâmicas de operação com a conversão do $\mathrm{SO}_{2}$. Para tanto, este trabalho utilizou uma planta piloto de $250 \mathrm{~kW}_{\mathrm{t}}$ em leito fluidizado borbulhante para a análise da conversão do $\mathrm{SO}_{2}$ a $\mathrm{CaSO}_{4}$ com dois distintos tipos de carvão mineral. Foi realizada uma análise estequiométrica e fluidodinâmica do processo a partir de dados obtidos da emissão de $\mathrm{SO}_{2}$ nos gases de combustão. A \% de conversão de $\mathrm{SO}_{2}$ chegou a 70,5\% para um carvão utilizado e 59,5\% para outro carvão. O maior teor de enxofre de um dos carvões mostrou influência na extensão da reação, especificamente no número de mols de $\mathrm{SO}_{2}$ consumidos por hora. A análise mineralógica mostrou que ocorre uma das rotas descritas na literatura com formação intermediária de $\mathrm{CaSO}_{3}$ por duas reações em série e a elutriação das cinzas e do $\mathrm{CaO}$ mostraram distintas tendências.
\end{abstract}

\section{INTRODUÇÃO}

A dessulfuração in situ, em leito fluidizado borbulhante, pela reação do $\mathrm{CaO}$ presente no calcário com o $\mathrm{SO}_{2}$ gerado em reações de combustão, está longe de ser estequiométrica, utilizando excessos de $\mathrm{Ca}$ em base molar de 200 a 300\%. Consequentemente, tal necessidade de excesso vem a ser uma das principais limitações desta tecnologia (Anthony a Granatstein, 2001).

Isto ocorre porque a formação do $\mathrm{CaSO}_{4}$ gera uma casca ao redor do $\mathrm{CaO}$ não reagido, de maior volume molar, que reduz a sua fragmentação (Scala et al., 2011). Algumas condições operacionais, como influência da temperatura e excesso de oxigênio já foram extensivamente descritas na literatura tendo como base alguns autores específicos (Yates, 1983; Ulerich et al., 1980). No entanto, relações entre as condições fluidodinâmicas - especificamente a elutriação - e a dessulfuração ainda possuem espaço para maior investigação, uma vez que o carregamento de sólidos para fora do sistema é fortemente afetado pela velocidade do gás e finos no leito (Kunii e Levenspiel, 1991).

Alguns autores têm estudado a influência das condições operacionais na dessulfuração, como 
Tarelho et al. (2005), que utilizaram dois tipos de carvão e uma razão molar $\mathrm{Ca} / \mathrm{S}$ de 3,5, além de excesso de ar de 10, 25 e 50\%. Apesar de alguma influência do excesso de ar, a alimentação de ar em estágio mostrou muito maior influência, o que propiciou maior conversão. Montanagro et al. (2010) investigaram a influência da temperatura no atrito e fragmentação de dois tipos de calcário na dessulfuração em um reator de leito fluidizado. A elutriação ocorreu mais fortemente durante a sulfatação e, após a formação da casca de $\mathrm{CaSO}_{4}$, o atrito passou a dominar no sistema. Ainda, Scala et al. (2011) descreveram a fragmentação de dois tipos de calcário e Hlincik e Buryan (2013) descreveram a performance de diferentes tipos de calcário, com diferentes teores de $\mathrm{CaO}$.

Para este trabalho, foi utilizado um tipo de calcário e dois tipos de carvão mineral, todos da Região Sul do Brasil, para a dessulfuração em planta piloto com reator de $250 \mathrm{~kW}_{\mathrm{t}}$ de leito fluidizado borbulhante. As conversões de $\mathrm{SO}_{2}$ foram obtidas para diferentes razões molares $\mathrm{Ca} / \mathrm{S}$ empregadas 1,5, 2,0 e 2,5. A análise dos resultados foi realizada pela obtenção dos rendimentos, extensão da reação, composição das cinzas e obtenção da elutriação das cinzas e $\mathrm{CaO}$. Com isso, uma relação da estequiometria das reações e das condições fluidodinâmicas foi possível de ser desenvolvida.

\section{METODOLOGIA}

\subsection{Materiais}

Segue, na Tabela 1, a caracterização dos carvões utilizados como combustíveis. Os carvões foram, ao longo do texto, chamados de "Carvão A" e "Carvão B". O calcário utilizado possui, quanto aos óxidos majoritários, $24,5 \% \mathrm{CaO}, 20,0 \% \mathrm{MgO}$ e 23,8\% $\mathrm{SiO}_{2}$, além da perda ao fogo de 28,0\% e Diâmetro de Sauter de 142,2 $\mu \mathrm{m}$.

Tabela 1 - Caracterização dos combustíveis.

\begin{tabular}{|c|c|c|}
\hline Carvão & A & B \\
\hline $\mathbf{C ~ ( \% ) ~}$ & 35,90 & 36,59 \\
\hline $\mathbf{H}(\%)$ & 2,78 & 2,57 \\
\hline $\mathbf{S}(\%)$ & 1,33 & 1,71 \\
\hline $\mathbf{N}(\%)$ & 0,63 & 1,17 \\
\hline $\mathbf{O}(\%)$ & 5,16 & 7,79 \\
\hline Cinzas (\%) & 45,67 & 40,17 \\
\hline PCI (kcal/kg) & 3813,33 & 3746,35 \\
\hline
\end{tabular}

\subsection{Planta piloto}

A Figura 1 mostra o esquema da planta piloto de $0,25 \mathrm{MW}_{\mathrm{t}}$ utilizada nas operações de combustão sem e com dessulfuração.

A planta consiste da alimentação de combustível e calcário, armazenados em silos (1) e (3), no reator em leito fluidizado borbulhante (6), por roscas dosadoras (2) e (4). O ar comburente é alimentado a partir de um ventilador (20). Os gases gerados na combustão passam pelo Ciclone 1 
(7), onde boa parte das cinzas é abatida e armazenada no Silo 1 (8). Os gases ainda passam pelo Ciclone 2 (11) antes de sair pela chaminé (12).

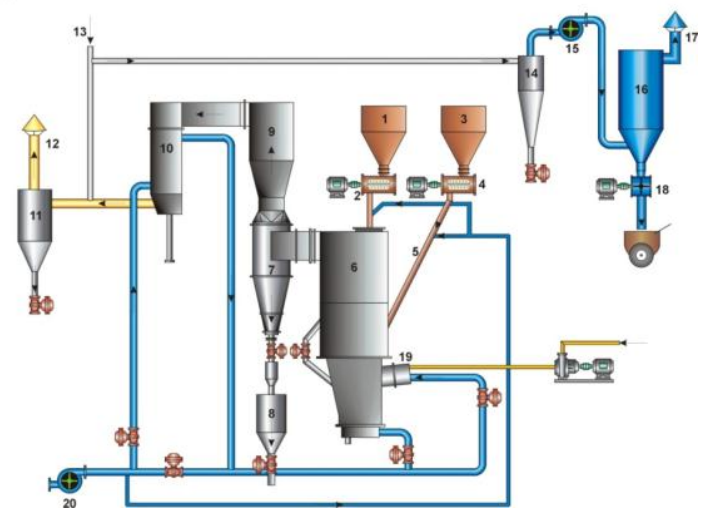

Figura 1 - Esquema da planta piloto em leito fluidizado borbulhante.

\subsection{Análise estequiométrica}

Para a dessulfuração, duas rotas, usualmente descritas na literatura, foram consideradas para a reação do $\mathrm{CaO}$ do calcário com o $\mathrm{SO}_{2}$ gerado na combustão. A primeira rota - Rota I considera a formação intermediária de $\mathrm{CaSO}_{3}$ e, posteriormente, a sua oxidação a $\mathrm{CaSO}_{4}$ (Yates, 1983; Anthony e Granatstein, 2001). A segunda rota - Rota II - já considera a formação direta do $\mathrm{CaSO}_{4}$ (Suyadal et al., 2005).

Extensão da reação: A extensão da reação, $\xi$, denota o quanto da reação está ocorrendo e foi determinada pela Equação 1 (Himmenblau e Riggs, 2006):

$$
\xi=\frac{\operatorname{SO}_{2}\left(\text { sai) }-S O_{2}(\text { entra })\right.}{-1}
$$

\% Conversão $\mathrm{SO}_{2}:$ A Equação 2 mostra a expressão para a determinação da conversão do $\mathrm{SO}_{2}$ gerado.

$$
\% \text { Conversão } \mathrm{SO}_{2}=\frac{n_{\tilde{\mathrm{iOSO}} \mathrm{z}}-n_{\mathrm{iSO}}}{n_{\mathrm{ioSO}}} \times 100
$$

Rendimento da dessulfuração: Para a determinação do rendimento de $\mathrm{CaSO}_{4}$, utilizou-se a definição do mesmo baseado na alimentação (Himmenblau e Riggs, 2006), sendo a quantidade obtida de produto desejado - $\mathrm{CaSO}_{4}$ - dividida pela quantidade alimentada do reagente.

\subsection{Elutriação}

Foram determinadas as \% Elutriação de Cinzas e \% Elutriação $\mathrm{CaO}$, sendo que a massa elutriada foi determinada pela massa depositada no Silo 1 (Figura 1). 


\section{RESULTADOS E DISCUSSÃO}

\subsection{Resultados da combustão, dessulfuração e caracterização das cinzas quanto aos óxidos}

A Tabela 2 apresenta duas diferentes condições aplicadas para a combustão do Carvão A e Carvão B. Para cada carvão, foi utilizada uma velocidade superficial $\left(U_{\mathrm{s}}\right)$ distinta, de $0,9 \mathrm{~m} / \mathrm{s}$ e $1,2 \mathrm{~m} / \mathrm{s}$, tendo a reação já em estado estacionário e as temperaturas abaixo (T04) e acima (T05) do leito fluidizado entre 850 e $870^{\circ} \mathrm{C}$. Consequentemente, 4 condições distintas de processo foram obtidas: A1 e A2 para o Carvão A e B1 e B2 para o carvão B. A Tabela 3 já apresenta os resultados da dessulfuração para as duas velocidades superficiais empregadas e, para cada velocidade superficial, o uso três excessos de cálcio.

Tabela 2 - Combustão em leito fluidizado borbulhante para o Carvão A e o Carvão B.

\begin{tabular}{|c|c|c|c|c|}
\hline Carvão & A & A & B & B \\
\hline Condição & A1 & A2 & B1 & B2 \\
\hline Razão Ar/Combustível & 6,5 & 8,7 & 8,0 & 9,1 \\
\hline $\mathbf{U}_{\mathbf{s}}(\mathbf{m} / \mathbf{s})$ & 0,9 & 1,2 & 0,9 & 1,2 \\
\hline $\mathbf{T 0 5}\left({ }^{\circ} \mathbf{C}\right)$ & 860 & 855 & 860 & 870 \\
\hline $\left.\mathbf{T 0 4}^{\circ} \mathbf{C}\right)$ & 859 & 856 & 855 & 865 \\
\hline $\mathbf{S O}_{2}(\mathbf{p p m})$ & 1084,7 & 976,2 & 943,7 & 776,1 \\
\hline
\end{tabular}

Como observado na Tabela 3, há uma diferença da conversão de $\mathrm{SO}_{2}$ entre $\mathrm{U}_{\mathrm{s}}=0,9 \mathrm{~m} / \mathrm{s}$ e $\mathrm{U}_{\mathrm{s}}=1,2 \mathrm{~m} / \mathrm{s}$, como esperado, de maneira a obter melhores condições para a menor velocidade superficial. Ao mesmo tempo, de maneira geral, o Carvão A mostrou melhores condições quanto à conversão de $\mathrm{SO}_{2}$.

Tabela 3 - Dessulfuração do Carvão A para $\mathrm{U}_{\mathrm{s}}=0,9 \mathrm{~m} / \mathrm{s}$ e $1,2 \mathrm{~m} / \mathrm{s}$.

\begin{tabular}{|c|c|c|c|c|c|c|}
\hline Condição & A3 & A4 & A5 & A6 & A7 & A8 \\
\hline $\mathbf{U}_{\mathrm{s}}(\mathbf{m} / \mathbf{s})$ & \multicolumn{3}{|c|}{$U_{\mathrm{s}}=0,9 \mathrm{~m} / \mathrm{s}$} & \multicolumn{3}{|c|}{$U_{s}=1,2 \mathrm{~m} / \mathrm{s}$} \\
\hline Razão Molar Ca/S & 1,5 & 2,0 & 2,5 & 1,5 & 2,0 & 2,5 \\
\hline \% Conversão $\mathrm{SO}_{2}$ & 52,5 & 62,5 & 70,5 & 44,7 & 45,7 & 60,9 \\
\hline Condição & B3 & B4 & B5 & B6 & B7 & B8 \\
\hline \% Conversão $\mathrm{SO}_{2}$ & 37,0 & 51,8 & 59,5 & 28,8 & 48,0 & 47,4 \\
\hline
\end{tabular}

\subsection{Análise estequiométrica}

Tendo em conta os resultados obtidos a partir das Tabelas 2 e 3, a Tabela 4 mostra o rendimento de $\mathrm{CaSO}_{4}$ obtido em relação à alimentação de $\mathrm{SO}_{2}$ e $\mathrm{CaO}$. Ao mesmo tempo, a Tabela 4 também mostra a extensão da reação quanto ao número de mols de $\mathrm{SO}_{2}$ consumidos.

Considerando os rendimentos de mol de $\mathrm{CaSO}_{4}$ por mol de reagente, são observadas diferenças nas comparações A4 x A7 e B4 x B7. Para um mesmo tipo de carvão, o número de 
mols de $\mathrm{CaSO}_{4}$ formado por mol de $\mathrm{SO}_{2}$ e $\mathrm{CaO}$ consumidos foi maior quando houve o uso de uma menor velocidade superficial. Aqui já se observa uma tendência de maior consumo de reagentes para um maior tempo de residência, como esperado.

Tabela 4: Estequiometria da dessulfuração quanto ao rendimento de $\mathrm{CaSO}_{4}$ e extensão da reação.

\begin{tabular}{|c|c|c|c|}
\hline Condição & mols CaSO $/ \mathbf{m o l s ~} \mathbf{S O}_{\mathbf{2}}$ feed & mols CaSO $/ \mathbf{m o l s ~ C a O ~ f e e d ~}$ & $\boldsymbol{\xi}\left(\mathbf{n}^{\circ} \mathbf{m o l s} / \mathbf{h}\right)$ \\
\hline A4 & 0,625 & 0,333 & $-7,29$ \\
\hline B4 & 0,518 & 0,322 & $-10,35$ \\
\hline A7 & 0,457 & 0,225 & $-7,98$ \\
\hline B7 & 0,480 & 0,273 & $-8,79$ \\
\hline
\end{tabular}

Para uma comparação das condições A4 x B4 e A7 x B7, isto é, para mesmas condições fluidodinâmicas, porém carvões diferentes, há questões a serem consideradas. Especialmente, verificam-se valores distintos na extensão da reação. Mesmo com menor conversão de $\mathrm{SO}_{2}$ para o Carvão B nas condições A4 x B4 e conversão similar nas condições A7 x B7, o Carvão B apresentou uma maior extensão da reação quanto ao consumo de $\mathrm{SO}_{2}$, em mols/h. Este comportamento vem a ser consequência do maior teor de enxofre no Carvão B, o qual, apesar de apresentar menor \% de dessulfuração, conseguiu manter maior taxa de reação. Além disso, para o mesmo carvão, as condições fluidodinâmicas mostraram uma influência significativa, uma vez que, para uma velocidade superficial de $0,9 \mathrm{~m} / \mathrm{s}$ a extensão da reação foi de $-10,35 \mathrm{mols} \mathrm{SO}_{2} / \mathrm{h}$ na condição $\mathrm{B} 4 \mathrm{e}-8,79$ mols $\mathrm{SO}_{2} / \mathrm{h}$ na condição $\mathrm{B} 7$.

\subsection{Análise mineralógica das cinzas}

Os difratogramas da análise mineralógica por Difração de Raios X seguem nas Figuras 2, sem dessulfuração e com dessulfuração, para as duas $\mathrm{U}_{\mathrm{s}}$ utilizadas, com Razão Molar Ca/S de 2,0 .

Observou-se nas cinzas A4, A7 e B4 a presença de sulfito de cálcio $\left(\mathrm{CaSO}_{3}\right)$. Em uma primeira análise, não é possível relacionar a formação de tal composto com a condição fluidodinâmica, uma vez que, nas respectivas cinzas, foram aplicadas velocidades superficiais de 0,9 e 1,2 m/s. De qualquer maneira, segundo Dam-Johansen and Ostergaard (1991), a formação de $\mathrm{CaSO}_{4}$ a partir de $\mathrm{CaO}$ e $\mathrm{SO}_{2}$, com oxigênio no sistema, pode ocorrer tanto pela formação inicial de $\mathrm{CaSO}_{3}$ e subsequente oxidação a $\mathrm{CaSO}_{4}$, ou oxidação direta a $\mathrm{CaSO}_{4}$ a partir da formação de $\mathrm{SO}_{3}$ a partir do $\mathrm{SO}_{2}$ e subsequente reação com o CaO. Tais considerações, a partir dos resultados, sugerem que, para o leito fluidizado borbulhante, pelo menos para o Carvão A a rota para a dessulfuração do $\mathrm{SO}_{2}$ com calcário dolomítico ocorre pela formação intermediária do $\mathrm{CaSO}_{3}$, como proposto na Rota I.

Mais ainda, ao longo da operação de dessulfuração, foi observado nos difratogramas a formação de Monticellite $\left(\mathrm{CaMgSiO}_{4}\right)$, provavelmente oriundo da interação da dolomita, $\mathrm{CaMg}\left(\mathrm{CO}_{3}\right)_{2}$ com a sílica presente no leito, o que vem a ser um resultado ainda não descrito na literatura.. 

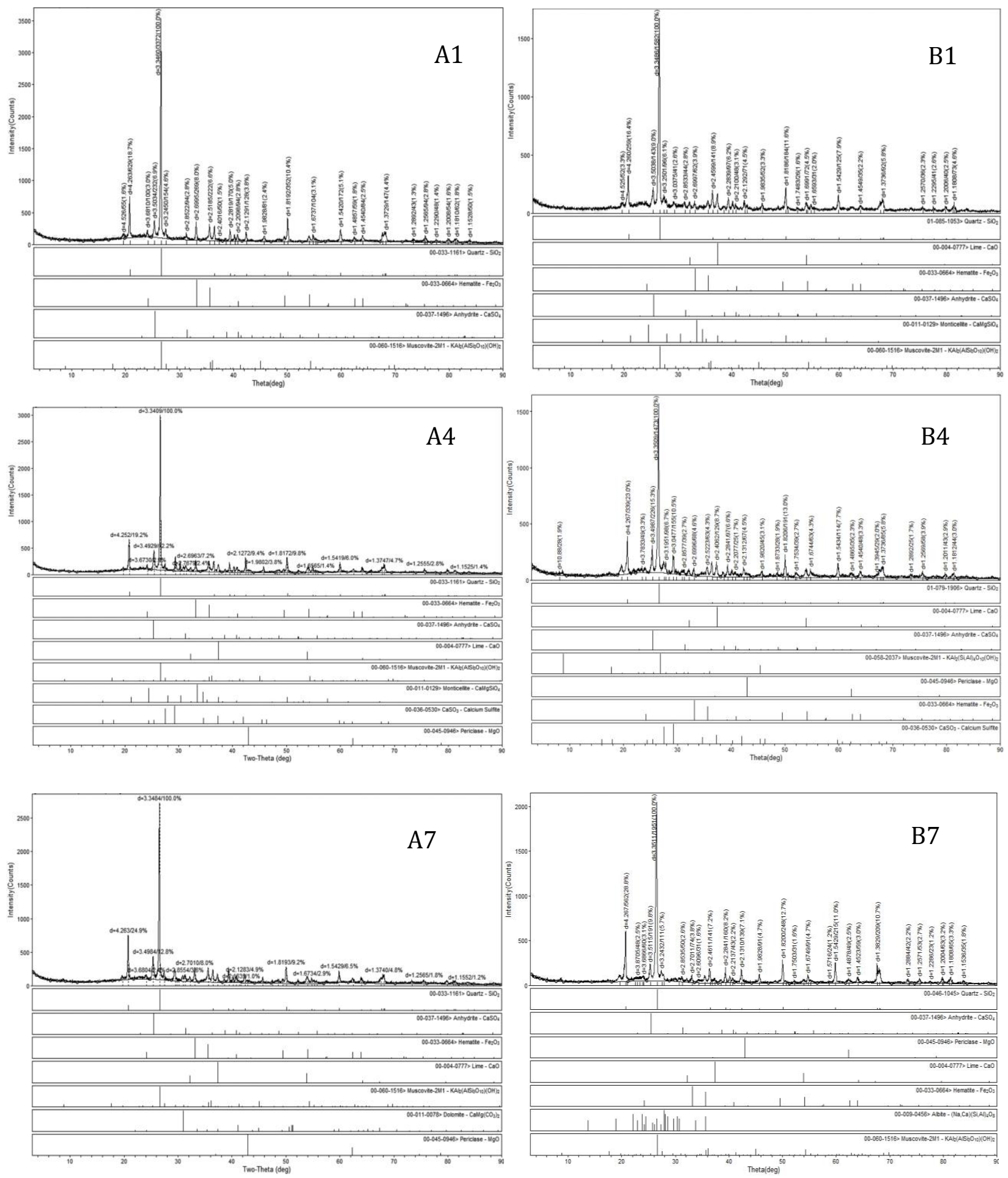

Figura 2: Difratogramas das cinzas Sem dessulfuração (A1 e B1); Com Dessulfuração Ca/S 2.0 e $\mathrm{U}_{\mathrm{s}}=0.9 \mathrm{~m} / \mathrm{s}\left(\mathrm{A} 4\right.$ e B4), e Com Dessulfuração Ca/S 2.0 e $\mathrm{U}_{\mathrm{s}}=1.2 \mathrm{~m} / \mathrm{s}(\mathrm{A} 7$ e B7). 


\subsection{Elutriação}

A Tabela 5 apresenta a \% de cinzas obtidas a partir do Ciclone 1 nas condições A4 e A7 assim como a porcentagem de $\mathrm{CaO}$ a partir do mesmo ciclone.

Tabela 5: Elutriação do Carvão A para a Razão Molar Ca/S 2,0 e $\mathrm{U}_{\mathrm{s}}=0,9 \mathrm{~m} / \mathrm{s}$ and 1,2 m/s.

\begin{tabular}{|c|c|c|}
\hline Condição & A4 & A7 \\
\hline \% Cinza Silo 1 & 60,5 & 55,4 \\
\hline \% CaO Silo 1 & 43,07 & 37,32 \\
\hline
\end{tabular}

Os valores da Tabela 5 sugerem que a elutriação para as cinzas e o $\mathrm{CaO}$ proveniente do calcário tende a ser distinta. Primeiramente, deve ser comentado que a \% tanto de cinzas quanto de $\mathrm{CaO}$ presente no Silo 1, proveniente do Ciclone 1, foi levemente menor para a condição A7, quando houve maior velocidade de entrada de ar. Este vem a ser um valor distinto do normalmente esperado. Usualmente, tem-se maior elutriação para uma maior velocidade superficial. Com isso, tem-se a hipótese de que tais porcentagens elutriadas e abatidas no Ciclone 1 vêm a ser as máximas obtidas para este equipamento durante a operação nas condições de fluidização borbulhante, uma vez que chegou a ser observada a presença de leito elutriado nas cinzas obtidas da condição A7. Além disso, em tais condições, a visualização do leito mostrou uma condição turbulenta.

Consequentemente, pergunta-se se o restante das cinzas e do $\mathrm{CaO}$ ficou mais concentrado no leito ou, por fragmentação, não foram possíveis de serem abatidos pelo Ciclone 1 (e também pelo Ciclone 2, onde não foi observado abatimento). Na Figura 3, observam-se, com exceção da Hematita, os produtos oriundos da adição de calcário dolomítico e da dessulfuração in situ. Tais resultados sugerem que tais produtos da dessulfuração, se não abatidos pelo Ciclone $1 \mathrm{e}$ armazenados no Silo 1, tendem a se concentrar no leito, o que pode explicar a menor concentração de $\mathrm{CaO}$ no Silo 1 em comparação com as cinzas como mostrado na Tabela 6.

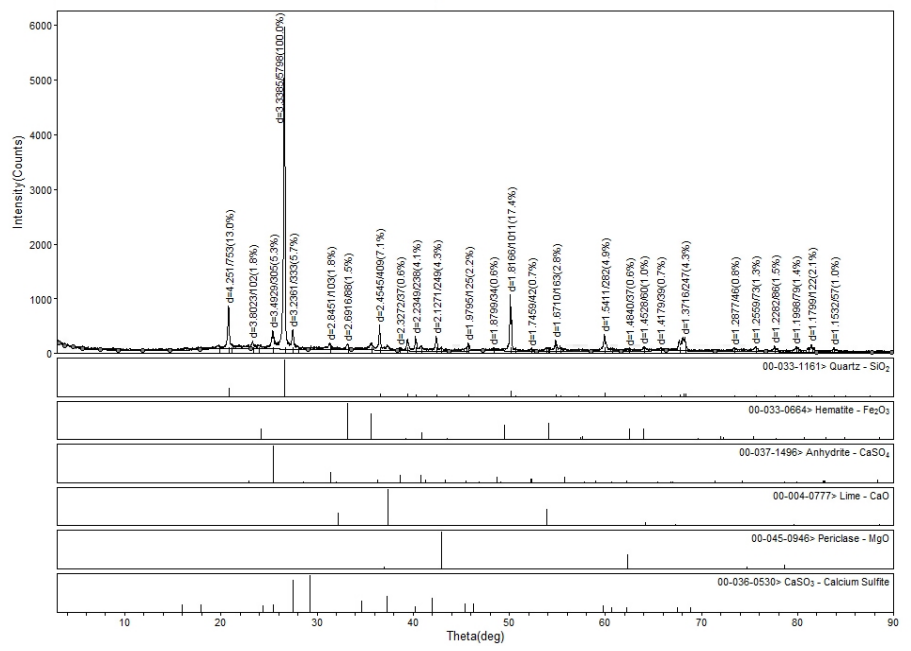

Figura 3: Difratograma do leito após a operação com o Carvão A. 


\section{CONCLUSÕES}

Quanto à análise estequiométrica, o maior valor da extensão da reação para as duas velocidades superficiais do Carvão B deve ser salientado, apesar de apresentar menor \% de dessulfuração, conseguindo manter maior taxa de reação. A análise mineralógica mostrou a presença, além dos produtos usuais da dessulfuração em leito fluidizado, a presença de $\mathrm{CaSO}_{3}$. A formação de tal composto sugere que a rota de dessulfuração ocorre por duas reações em série, tendo a formação de $\mathrm{CaSO}_{3}$ como um composto intermediário. Observou-se, claramente, uma menor concentração de $\mathrm{CaO}$ elutriado em comparação com as cinzas. Tais valores foram explicados pela tendência de concentração dos produtos da dessulfuração $-\mathrm{CaSO}_{4}, \mathrm{CaO}, \mathrm{CaSO}_{3}$ - no leito após a dessulfuração.

\section{REFERÊNCIAS}

ANTHONY, E.J.; GRANATSTEIN, D.L. Sulfation phenomena in fluidized combustion systems. Progress. in En. and Comb. Sci., v. 27, p. 215-236, 2001.

DAM-JOHANSEN, K.; OSTERGAARD, K. High-temperature between sulphur dioxide and limestone - IV. A discussion of chemical reaction mechanism and kinetics. Chem. Eng. Sci., v. 46, p. 588-859, 1991.

HIMMELBLAU, D.M.; RIGGS, J.B. Basic Principles and Calculations in Chemical Engineering. Rio de Janeiro: Pearson, 2006.

HLINCIK, T.; BURYAN, P. Evaluation of limestones for the purposes of desulphurization during the fluid combustion of brown coal. Fuel, v. 104, p. 208-215, 2013.

KUNII, D.; LEVENSPIEL, O. Fluidization Engineering. Londres: Butterworths, 1991.

MONTANGRO, F.; SALATINO, P., SCALA, F. The influence of temperature on limestone sulfation and attrition under fluidized bed combustion conditions. Exp. Thermal and Fluid Sci., v. 34, p. 352-358, 2010.

SCALA, F.; LUPÍAÑEZ, C.; SALATINO, P.; ROMEO, L.M.; DÍEZ, L.I. Primary fragmentation of limestone under oxy-firing. Fuel Proc. Tech., v. 92, p. 1449-1456, 2011.

SUYADAL, Y.; EROL, M.; OGUZ, H. Deactivation model for dry desulphurization of simulated flue gas with calcined limestone in a fluidized-bed reactor. Fuel, v. 84, p. 1705-1712, 2005.

TARELHO, L.A.C; MATOS, M.A.A.; PEREIRA, F.J.M.A. The influence of operational parameters on $\mathrm{SO}_{2}$ removal by limestone during fluidized bed combustion. Fuel Proc. Tech., v. 86, p. 1385-1401, 2005

ULERICH, N.H.; NEWBY, R.A.; KEAIRNS, D.L. A thermogravimetric study of the sulfation of limestone and dolomite - Prediction of pressurized and atmospheric fluidized bed desulfurization. Thermoch. Acta, v. 36, 1-16, 1980.

YATES, J.G. Fundamentals of Fluidized-Bed Chemical Processes. Londres: Butterworths, 1983. 